\title{
Preface to the August 2018 Issue including selected works from CLEI 2017 plus one regular paper
}

In this special issue a selection of the best papers presented during the 42th Latin American Conference in Informatics (CLEI 2017) is presented in extended versions. After a very tough selection process for the Conference, a set of 10 paper obtained the best review marks. The authors were invited to submit extended versions that were again blind-reviewed, and finally eight were accepted for publication. Considering that over 400 papers were originally submitted to the conference for evaluation, this selection reflects how competitive is the process.

In the first paper, "Towards Autonomous Reinforcement Learning: Automatic Setting of Hyper-parameters using Bayesian Optimization", the authors introduce a framework based on Bayesian optimization with Gaussian process regression to optimize the hyper-parameters of a reinforcement learning algorithm. This framework may be used in different areas from text mining to autonomous car driving.

The second paper, "Maximum Diversity Problem. A Multi-Objective Approach", presents for the first time multi-objective approaches for the MD problem, considering the simultaneous optimization of the following five diversity measures: (i) Max-Sum, (ii) Max-Min, (iii) Max-MinSum, (iv) Min-Diff and (v) Min-P-center. The auhors propose two approaches for this, one based on the Multi-objective Maximum Diversity, and the other on Multi-objective Average Diversity. In both cases an evolutionary algorithm is presented to solve the optimization problem.

The third paper is "A formal approach for the verification of the permissionbased security model of Android". In it, the authors have developed a comprehensive formal specification of Android's permission model, which has been used to state and prove properties that establish expected behavior of the procedures that enforce the defined access control policy. They describe the formalized model, present security properties that have been proved using the Coq proof assistant and propose the use of a certified algorithm for performing verification activities such as monitoring of actual implementations of the platform and also as a testing oracle.

In the fourth paper, "A Model-Driven Approach to develop Rich Web Applications", the authors introduce Model Driven Develpoment techniques to the web application development by defining a specific architecture model with Rich Internet Applications functionalities. For this, the necessary metamodels and UML profiles are defined as well as the transformation rules to allow an easy code generation in different languages. 
The fifth paper, "Data Quality Management in e-Health Integration Platforms: the Case of Uruguay" proposed a solution for data quality management in the context of the Uruguayan e-health integration platform. The proposal comprises a data quality model, that includes quality characteristics identified from different types of sources, and extensions to an ESB-based integration platform which allows assessing and enforcing data quality requirements in inter-organizational data exchanges.

In the sixth paper, "Collaborative Business Process Management Through a Platform Based on Cloud Computing", the authors propose a cloud-based platform for the management of Collaborative Business Processes (CBP). The platform provides cloud services that enable the generation and deployment on-demand of the Process-Aware Information Systems required to implement the agreed CBPs, as well as the execution on-demand of CBPs by fulfilling the abovementioned issues. Besides attention to privacy, elasticity, and portability issues are offered.

The seventh paper, "Systematic evaluation of Business Process Management Systems", presents a methodology to evaluate systematically BPMS ensuring quality of the results and the repeatability of the evaluation with a detailed definition of non-functional aspects to be evaluated, with a set of test cases for their evaluation and the development of a case-study.

In the eighth paper, "Simple Energy Aware Scheduler: An Empirical Evaluation", the authors present a distributed computing platform for mobile devices that support Simple Energy Aware Scheduler (SEAS) and an empirical evaluation of the SEAS scheduler. This evaluation followed the methodology of the original SEAS evaluation, in which Random and Round Robin schedulers were used as baselines. Although SEAS has been proposed in 2010, this is the first time that an actual implementation is evaluated in real mobile devices.

The last paper, “The 'Code Yourself!' and ' $\mathrm{A}$ A Programar!' programming MOOC for teenagers: Reflecting on one and a half years of experience", is a regular paper which completes the current issue. In this work, the authors describe their experience in creating and maintaining a MOOC course for young teenagers who want to learn programming skills. The MOOC course, available in English and Spanish language, was delivered in different modes, and attained a large number of students. The authors discuss the characteristics of the MOOC and analyze the results obtained and the level of expectations attainment by the students.

Dr. Rodrigo Santos - Invited Editor

Dep. Ingenieria Electrica y de Computadoras

Universidad Nacional del Sur

Instituto de Ciencias e Ingenieria e Computacion

CONICET 